\title{
On the Euler superintegrable systems
}

\author{
Yu A Grigoryev, V. A. Khudobakhshov, A V Tsiganov \\ St.Petersburg State University, St.Petersburg, Russia \\ e-mail: com974@mail.ru,vitaly.khudobakhshov@gmail.com,tsiganov@mph.phys.spbu.ru
}

\begin{abstract}
We discuss the Euler approach to construction and to investigation of the superintegrable systems with additional quadratic and cubic integrals of motions.
\end{abstract}

PACS numbers: 02.30.Jr, 02.30.Ik, 03.65.Fd

Mathematics Subject Classification: 70H06, 70H20, 35Q72

\section{Introduction}

The story began in 1761 when Euler investigated equations

$$
\frac{\mathrm{dx}_{1}}{\sqrt{P\left(\mathrm{x}_{1}\right)}} \pm \frac{\mathrm{dx}_{2}}{\sqrt{P\left(\mathrm{x}_{2}\right)}}=0
$$

where $P$ is an arbitrary quartic. Solving this problem Euler found additional algebraic integral of these equations and proposed an algebraic construction of the corresponding classical trajectories of motion without any integration and inversion of the Abel map [6].

The Euler results were generalized by Abel, Jacobi, Lagrange, Weierstrass and many other. Here we mention works of Richelot [11] and Weierstrass [16], who found generating function of additional integrals of motion in the $n$-dimensional case.

The main aim of this note is to discuss this oldest but almost completely forgotten in modern literature Euler's approach to construction and to investigations of the superintegrable systems.

\section{The Euler results}

The first demonstration of the existence of an addition theorem for elliptic functions is due to Euler [6], who showed that the differential relation

$$
\frac{\mathrm{dx}}{\sqrt{X}}+\frac{\mathrm{dy}}{\sqrt{Y}}=0
$$

connecting the most general quartic function of a variable $x$

$$
f=a \mathrm{x}^{4}+4 b \mathrm{x}^{3}+6 c \mathrm{x}^{2}+4 d \mathrm{x}+e
$$

and the same function $Y$ of another variable y, leads to an algebraical relation between $\mathrm{x}$ and $\mathrm{y}, X$ and $Y$ :

$$
\left(\frac{\sqrt{X}-\sqrt{Y}}{\mathrm{x}-\mathrm{y}}\right)^{2}=a(\mathrm{x}+\mathrm{y})^{2}+4 b(\mathrm{x}+\mathrm{y})+C
$$

where $C$ is the arbitrary constant of integration. This algebraic relation when rationalized leads to a symmetrical biquadratic form of $\mathrm{x}$ and $\mathrm{y}$

$$
F(\mathrm{x}, \mathrm{y})=a \mathrm{x}^{2} \mathrm{y}^{2}+2 b \mathrm{xy}(\mathrm{x}+\mathrm{y})+c\left(\mathrm{x}^{2}+4 \mathrm{xy}+\mathrm{y}^{2}\right)+2 d(\mathrm{x}+\mathrm{y})+e=0,
$$


which defines the conic section on the plane $(\mathrm{x}, \mathrm{y})$, which then will be classical trajectory of motion in the configurational space.

According to [2] we could replace the constant $C$ in the Euler integral relation by $4 c+4 s$, where

$$
s=\frac{F(\mathrm{x}, \mathrm{y})-\sqrt{X} \sqrt{Y}}{2(\mathrm{x}-\mathrm{y})^{2}}=\frac{1}{4}\left(\frac{\sqrt{X}-\sqrt{Y}}{\mathrm{x}-\mathrm{y}}\right)^{2}-\frac{a(\mathrm{x}+\mathrm{y})^{2}}{4}-b(\mathrm{x}+\mathrm{y})-c,
$$

is the famous algebraic Euler integral. Treating $s$ as a function of the independent variables $\mathrm{x}$ and $\mathrm{y}$, one gets the following addition theorem

$$
\frac{\mathrm{dx}}{\sqrt{X}}+\frac{\mathrm{dy}}{\sqrt{Y}}+\frac{\mathrm{d} s}{\sqrt{S}}=0
$$

Of course, the Euler addition theorem is a very special case of the Abel theorem [2].

The polynomial $S$ in (2.5) may be defined in the algebraic form

$$
\sqrt{S}=\frac{\left(Y_{1} \mathrm{x}+Y_{2}\right) \sqrt{X}-\left(X_{1} \mathrm{y}+X_{2}\right) \sqrt{Y}}{(\mathrm{x}-\mathrm{y})^{3}}
$$

where

$$
X_{1}=\left(a \mathrm{x}^{3}+3 b \mathrm{x}^{2}+3 c \mathrm{x}+d\right), \quad X_{2}=b \mathrm{x}^{3}+3 c \mathrm{x}^{2}+3 d \mathrm{x}+e
$$

and similar to $Y_{1,2}$, or in the Weierstrass form

$$
S=4 s^{3}-g_{2} s-g_{3}
$$

where

$$
g_{2}=a e-4 b d+3 c^{2}, \quad g_{3}=a c e+2 b c d-a d^{2}-e b^{2}-c^{3},
$$

are the quadrivariant and cubicvariant of the quartic $X$ [1], respectively.

Changing the sign of $\sqrt{Y}$, we find that

$$
s=\frac{F(\mathrm{x}, \mathrm{y})+\sqrt{X} \sqrt{Y}}{2(\mathrm{x}-\mathrm{y})^{2}}
$$

leads to another differential relation

$$
\frac{\mathrm{dx}}{\sqrt{X}}-\frac{\mathrm{dy}}{\sqrt{Y}}+\frac{\mathrm{d} s}{\sqrt{S}}=0 .
$$

A more elegant expression can be given to these relations if we follow Klein [2] in employing homogeneous variables $\mathrm{x}_{1,2}$ and $\mathrm{y}_{1,2}$, by writing $\mathrm{x}_{1} / \mathrm{x}_{2}$ for $\mathrm{x}$ and $\mathrm{y}_{1} / \mathrm{y}_{2}$ for $\mathrm{y}$.

In generic case the differential equations

$$
\sum_{i=1}^{n} \frac{\mathrm{x}_{i}^{k} \mathrm{dx}_{i}}{\sqrt{X_{i}}}=0, \quad k=0,1, \ldots, n-2
$$

connecting polynomials

$$
X_{i}=a_{2 n} \mathrm{x}_{i}^{2 n}+a_{2 n-1} \mathrm{x}_{i}^{2 n-1}+\cdots+a_{1} \mathrm{x}_{i}+a_{0},
$$

of variables $\mathrm{x}_{i}$, have the following additional integral

$$
s=\mathrm{x}_{1}^{2} \ldots \mathrm{x}_{n}^{2}\left(\sum_{i=1}^{n} \frac{\sqrt{X_{i}}}{\mathrm{x}_{i}^{2} F^{\prime}\left(\mathrm{x}_{i}\right)}\right)^{2}-a_{1}\left(\frac{1}{\mathrm{x}_{1}}+\cdots+\frac{1}{\mathrm{x}_{n}}\right)-a_{0}\left(\frac{1}{\mathrm{x}_{1}}+\cdots+\frac{1}{\mathrm{x}_{n}}\right)^{2},
$$

where $F(\mathrm{x})=\prod\left(\mathrm{x}-\mathrm{x}_{j}\right)$ [11]. At $n=2$ this integral is related with the Euler integral (2.4), see [11]. Moreover, at $n>2$ we can get a whole family of such functionally independent additional integrals $[11,16]$. 


\section{Classification of the Euler superintegrable systems}

In order to use the Euler addition theorems (2.5) and (2.10) for construction of the superintegrable Stäckel systems we have to start with the genus one hyperelliptic curve

$$
\mu^{2}=P(\lambda), \quad \text { where } \quad P(\mathrm{x})=X,
$$

and a pair of arbitrary substitutions

$$
\lambda_{j}=v_{j}\left(q_{j}\right) \quad \mu_{j}=u_{j}\left(q_{j}\right) p_{j}, \quad j=1,2,
$$

where $p$ and $q$ are canonical variables $\left\{p_{j}, q_{i}\right\}=\delta_{i j}$.

This hyperelliptic curve and substitutions give us a pair of the separated relations

$$
p_{j}^{2} u_{j}^{2}\left(q_{j}\right)=a v_{j}\left(q_{j}\right)^{4}+4 b v_{j}\left(q_{j}\right)^{3}+6 c v_{j}\left(q_{j}\right)^{2}+4 d v_{j}\left(q_{j}\right)+e, \quad j=1,2,
$$

where coefficients $a, b, c, d$ and $e$ of the quartic $X(2.2)$ are linear functions of integrals of motion $H_{1,2}$ :

$$
a=\alpha_{1} H_{1}+\alpha_{2} H_{2}+\alpha, \quad b=\beta_{1} H_{1}+\beta_{2} H_{2}+\beta, \quad \ldots, \quad e=\epsilon_{1} H_{1}+\epsilon_{2} H_{2}+\epsilon .
$$

The separated relations (3.1) coincide with the Jacobi relations for the uniform Stäckel systems [12, $13,14]$

$$
p_{j}=\sqrt{\sum_{k=1}^{2} H_{k} \mathbf{S}_{k j}+U_{j}\left(q_{j}\right)}
$$

where $\mathbf{S}$ is the so-called Stäckel matrix and $U_{j}$ is the Stäckel potential:

$$
\begin{aligned}
& \mathbf{S}_{i j}=u_{j}^{-2}\left(\alpha_{i} v_{j}^{4}+4 \beta_{i} v_{1}^{3}+6 \gamma_{i} v_{j}^{2}+4 \delta_{i} v_{j}+\epsilon_{i}\right), \\
& U_{j}=u_{j}^{-2}\left(\alpha v_{j}^{4}+4 \beta v_{1}^{3}+6 \gamma v_{j}^{2}+4 \delta v_{j}+\epsilon\right),
\end{aligned}
$$

Solving these separated relations (3.1-3.2) with respect to $H_{1,2}$ one gets pair of the Stäckel integrals of motion in the involution

$$
H_{k}=\sum_{j=1}^{2}\left(S^{-1}\right)_{j k}\left(p_{j}^{2}-U_{j}\left(q_{j}\right)\right), \quad k=1,2,
$$

and angle variables

$$
\omega_{i}=\frac{1}{2} \sum_{j=1}^{2} \int \frac{\mathbf{S}_{i j} \mathrm{~d} q_{j}}{p_{j}}=\frac{1}{2} \sum_{j=1}^{2} \int \frac{\mathbf{S}_{i j} \mathrm{~d} q_{j}}{\sqrt{\sum_{k=1}^{2} H_{k} S_{k j}+U_{j}\left(q_{j}\right)}}
$$

canonically conjugated with the action variables $H_{1,2}$

$$
\left\{H_{1}, H_{2}\right\}=\left\{\omega_{1}, \omega_{2}\right\}=0, \quad\left\{H_{i}, \omega_{j}\right\}=\delta_{i j} .
$$

In generic case the action variables (3.4) are the sum of the multi-valued functions. However, if we are able to apply some addition theorem to the calculation of $\omega_{2}$

$$
\omega_{2}=\frac{1}{2} \int^{v_{1}\left(q_{1}\right)} \frac{\mathbf{S}_{21}(\lambda) \mathrm{d} \lambda}{\sqrt{P(\lambda)}}+\frac{1}{2} \int^{v_{2}\left(q_{2}\right)} \frac{\mathbf{S}_{22}(\lambda) \mathrm{d} \lambda}{\sqrt{P(\lambda)}}=\frac{1}{2} \int^{s} \frac{\mathrm{d} s}{\sqrt{S}},
$$

then one could get additional single-valued integrals of motion $s$ and $S$ :

$$
\left\{H_{1}, \omega_{2}\right\}=0 \quad \Rightarrow \quad\left\{H_{1}, s\right\}=\left\{H_{1}, S\right\}=0 .
$$


Of course, opportunity to apply some addition theorem to computation of the angle variable $\omega_{2}$ and of the additional single-valued integrals of motion $s, S$ leads to some restrictions on our quartic $P(\lambda)$ and substitutions $\lambda_{j}=v_{j}\left(q_{j}\right) \quad \mu_{j}=u_{j}\left(q_{j}\right) p_{j}$.

For instance, if we want to use the Euler addition theorems (2.5) and (2.10) we have to put

$$
\mathbf{S}_{21}(\lambda)=1, \quad \mathbf{S}_{22}(\lambda)= \pm 1
$$

Such as

$$
\mathbf{S}_{i j}(q)=\frac{v_{j}^{\prime}\left(q_{j}\right)}{u_{j}\left(q_{j}\right)} \mathbf{S}_{i j}(\lambda)
$$

these restrictions are equivalent to the following equations

$$
\kappa_{j} u_{j} v_{j}^{\prime}=\alpha_{2} v_{j}^{4}+4 \beta_{2} v_{j}^{3}+6 \gamma_{2} v_{j}^{2}+4 \delta_{2} v_{j}+\epsilon_{2}, \quad \kappa_{1}=1, \quad \kappa_{2}= \pm 1,
$$

on functions $u(q), v(q)$ and coefficients $\alpha_{2}, \beta_{2}, \ldots, \epsilon_{2}$ of the quartic, because we have to solve these equations in some fixed functional space [15].

It's easy to prove that there are five monomial solutions

$$
\begin{array}{llll}
\text { I } & u_{j}=1, & v_{j}=q_{j}, & \epsilon_{2} \neq 0 \\
\text { II } & u_{j}=q_{j}, & v_{j}=q_{j}^{4 \delta_{2} \kappa_{j}^{-1},} & \delta_{2} \neq 0, \\
\text { III } & u_{j}=1, & v_{j}=q_{j}^{-1}, & \gamma_{2} \neq 0 \\
\text { IV } & u_{j}=q_{j}^{-1}, & v_{j}=q_{j}^{-1}, & \beta_{2} \neq 0, \\
\text { V } & u_{j}=q_{j}^{-2}, & v_{j}=q_{j}^{-1}, & \alpha_{2} \neq 0
\end{array}
$$

up to canonical transformations of the separated variables $\left(p_{j}, q_{j}\right)$ and transformations of integrals of motion $H_{j} \rightarrow \sigma H_{j}+\rho$. Here notations $\alpha_{2} \neq 0, \beta_{2} \neq 0, \ldots$ mean that other parameters are equal to zero.

If we suppose that after some point transformation

$$
\begin{array}{ll}
x=z_{1}(q), & y=z_{2}(q), \\
p_{x}=\mathrm{w}_{11}(q) p_{1}+\mathrm{w}_{12}(q) p_{2}, & p_{y}=\mathrm{w}_{21}(q) p_{1}+\mathrm{w}_{22}(q) p_{2},
\end{array}
$$

with $\mathrm{w}_{i j} \neq 0$, kinetic part of the Hamilton function $H_{1}=T+V$ has a special form

$$
T=\sum\left(\mathbf{S}^{-1}\right)_{1 j} p_{j}^{2}=\mathrm{g}_{11}(x, y) p_{x}^{2}+\mathrm{g}_{12}(x, y) p_{x} p_{y}+\mathrm{g}_{22}(x, y) p_{y}^{2},
$$

where $\mathrm{g}$ is some fixed metric on a configurational manifold, then one gets some additional restrictions on the coefficients of the quartic $P(\lambda)$.

For instance, if we consider superintegrable systems on a complex Euclidean space $E_{2}(\mathbb{C})$

$$
T=\sum\left(\mathbf{S}^{-1}\right)_{1 j} p_{j}^{2}=p_{x} p_{y}
$$

then one gets the following algebraic equations

$$
\mathrm{w}_{11} \mathrm{w}_{21}=\left(\mathbf{S}^{-1}\right)_{11}, \quad \mathrm{w}_{12} \mathrm{w}_{21}+\mathrm{w}_{11} \mathrm{w}_{22}=0, \quad \mathrm{w}_{12} \mathrm{w}_{22}=\left(\mathbf{S}^{-1}\right)_{12}
$$

and the partial differential equations

$$
\left\{x, p_{x}\right\}=\left\{y, p_{y}\right\}=1, \quad\left\{p_{x}, y\right\}=\left\{p_{y}, x\right\}=\left\{p_{x}, p_{y}\right\}=0
$$

on coefficients $\alpha_{1}, \ldots \epsilon_{1}$ of the quartic and functions $z_{1,2}\left(q_{1}, q_{2}\right), \mathrm{w}_{k j}\left(q_{1}, q_{2}\right)$. The passage from the conformal coordinate system $(x, y)$ to another coordinate systems on the plain can be realized by using the Beltrami partial differential equations. 
The remaining free parameters $\alpha, \ldots, \epsilon$ determine potential part of the Hamiltonian $V(x, y)$. In fact, since integrals $H_{1,2}$ is defined up to the trivial shifts $H_{k} \rightarrow H_{k}+\rho_{k}$, our potential $V(x, y)$ depends on three arbitrary parameters only.

Summing up, we have proved that classification of the Euler superintegrable systems on the plain is equivalent to solution of the equations $(3.6,3.10,3.11)$. For all the possible solutions classical trajectory of motion is given by

$$
F\left(v_{1}\left(q_{1}\right), v_{2}\left(q_{2}\right)\right)=0
$$

see (2.3). Additional quadratic in momenta integral of motion looks like

$$
K_{2}=s+c=\frac{F\left(v_{1}, v_{2}\right)-p_{1} u_{1} p_{2} u_{2}}{2\left(v_{1}-v_{2}\right)^{2}}+c,
$$

see (2.4) and additional cubic in momenta integral of motion is equal to

$$
K_{3}=\sqrt{S} \equiv \sqrt{4 s^{3}-g_{2} s-g_{3}}
$$

see (2.6) and (2.7).

\subsection{Examples}

Let us find all the Euler superintegrable systems on a complex Euclidean space $E_{2}(\mathbb{C})$

$$
H_{1}=p_{x} p_{y}+V(x, y)
$$

with the real potentials $V$. Solving equations $(3.6,3.10,3.11)$ one gets the following five superintegrable potentials

$$
\begin{aligned}
& V_{1}=\alpha(x+y)+\beta(y+3 x) x^{-1 / 2}+\gamma x^{-1 / 2}, \\
& V_{2}=\alpha y\left(x+y^{2}\right)+\beta\left(x+3 y^{2}\right)+\gamma y, \\
& V_{3}=\alpha(x+3 y)(3 x+y)+\beta(x+y)+\frac{\gamma}{(x-y)^{2}} \\
& V_{4}=\alpha x y^{-3}-\beta y^{-2}-\gamma x y, \\
& V_{5}=\frac{\alpha}{x^{2}}+\frac{\beta}{x^{3 / 2} \sqrt{y-1}}+\frac{\gamma}{x^{1 / 2} \sqrt{y-1}} .
\end{aligned}
$$

Recall, that implicitly all these systems have been found by Euler in 1761 [6]. Potentials $V_{1}$ and $V_{3}$ in explicit form have been found by Drach [5], the $(\ell)$ and $(g)$ cases, whereas potential $V_{2}, V_{4}$ and $V_{5}$ may be found in [9], the $E_{10}, E_{8}$ and $E_{17}$ cases, respectively.

The first and fifth solutions (3.7) of the equations (3.6) are related with potentials $V_{1,2}$. The second and fourth solutions give us potential $V_{3}$, whereas third solution yields potentials $V_{4,5}$. Below we present some details of the calculations.

Case 1 If we take first solution from (3.7)

$$
u_{j}= \pm 1, \quad v_{j}= \pm q_{j}, \quad \Rightarrow \quad p_{1}^{2}=P\left(q_{1}\right), \quad\left(-p_{2}\right)^{2}=P\left(-q_{2}\right)
$$

and quartic

$$
P(\lambda)=-\frac{\alpha}{2} \lambda^{4}+2 \beta \lambda^{3}+H_{1} \lambda^{2}+2 \gamma \lambda+H_{2},
$$

then the Stäckel integrals are equal to

$$
\begin{aligned}
& H_{1}=\frac{p_{1}^{2}-p_{2}^{2}}{q_{1}^{2}-q_{2}^{2}}+\frac{\alpha\left(q_{1}^{2}+q_{2}^{2}\right)}{2}-\frac{2 \beta\left(q_{1}^{2}-q_{1} q_{2}+q_{2}^{2}\right)+2 \gamma}{q_{1}-q_{2}}, \\
& H_{2}=\frac{p_{2}^{2} q_{1}^{2}-p_{1}^{2} q_{2}^{2}}{q_{1}^{2}-q_{2}^{2}}-\frac{\alpha q_{1}^{2} q_{2}^{2}}{2}+\frac{\left(2 \beta q_{1} q_{2}+2 \gamma\right) q_{1} q_{2}}{q_{1}-q_{2}},
\end{aligned}
$$


whereas the second action variable looks like

$$
\omega_{2}=\frac{1}{2} \int^{q_{1}} \frac{\mathrm{dx}}{\sqrt{X}}-\frac{1}{2} \int^{-q_{2}} \frac{\mathrm{dy}}{\sqrt{Y}}=-\frac{1}{2} \int^{s} \frac{\mathrm{d} s}{\sqrt{S}} .
$$

Using addition theorem (2.10) we are able to get additional quadratic integral of motion

$$
K_{2}=s+c=\frac{1}{4}\left(\frac{p_{1}+p_{2}}{q_{1}+q_{2}}\right)^{2}+\frac{1}{8} \alpha\left(q_{1}-q_{2}\right)^{2}-\frac{1}{2} \beta\left(q_{1}-q_{2}\right)
$$

and additional cubic integral of motion (2.6)

$$
\begin{aligned}
K_{3}=\sqrt{S}= & -\frac{\left(p_{1}+p_{2}\right)^{2}\left(p_{1} q_{2}-p_{2} q_{1}\right)}{2\left(q_{1}+q_{2}\right)^{3}\left(q_{1}-q_{2}\right)}-\frac{\alpha\left(q_{1}-q_{2}\right)\left(p_{1} q_{2}-p_{2} q_{1}\right)}{4\left(q_{1}+q_{2}\right)}+\frac{\gamma\left(p_{1}+p_{2}\right)}{2\left(q_{1}^{2}-q_{2}^{2}\right)} \\
& -\frac{\beta\left(p_{1} q_{2}^{2}-2 p_{1} q_{1} q_{2}-2 p_{2} q_{1} q_{2}+p_{2} q_{1}^{2}\right)}{2\left(q_{1}^{2}-q_{2}^{2}\right)} .
\end{aligned}
$$

After the following change of variables

$$
x=\frac{\left(q_{1}-q_{2}\right)^{2}}{4}, \quad p_{x}=p_{1}-p_{2} q_{1}-q_{2}, \quad y=\frac{\left(q_{1}+q_{2}\right)^{2}}{2}, \quad p_{y}=\frac{p_{1}+p_{2}}{q_{1}+q 2},
$$

we obtain the Stäckel integrals

$$
\begin{aligned}
& H_{1}=p_{x} p_{y}+\alpha(x+y)+\frac{\beta(3 x+y)}{\sqrt{x}}+\frac{\gamma}{\sqrt{x}} \\
& H_{2}=\left(p_{x}-p_{y}\right)\left(p_{x} x-p_{y} y\right)-\frac{\alpha(x-y)^{2}}{2}-\frac{\beta(x-y)^{2}}{\sqrt{x}}+\frac{\gamma(x-y)}{\sqrt{x}},
\end{aligned}
$$

the Euler integrals

$$
\begin{aligned}
& K_{2}=s+c=\frac{p_{y}^{2}}{4}+\frac{\alpha x}{2}+\beta \sqrt{x} \\
& K_{3}=\sqrt{S}=\frac{p_{y}^{2}\left(p_{x}-p_{y}\right)}{4}+\frac{\alpha\left(p_{x}-p_{y}\right) x}{2}+\frac{\beta\left(2 p_{x} x-3 p_{y} x+p_{y} y\right)}{4 \sqrt{x}}+\frac{\gamma p y}{4 \sqrt{x}}
\end{aligned}
$$

and the following equation for the corresponding classical trajectory of motion

$$
F(x, y)=\left(x-\frac{y}{3}\right) H_{1}+H_{2}-\frac{\alpha(x-y)^{2}}{2}-2 \beta \sqrt{x}(x-y)-2 \gamma \sqrt{x}=0 .
$$

The same system may be obtained by using fifth substitution from (3.7).

Case 2 Using the same first solution (3.7) and another quartic

$$
P(\lambda)=-\frac{\alpha}{4} \lambda^{4}-\beta \lambda^{3}-\frac{\gamma}{2} \lambda^{2}+H_{1} \lambda+H_{2}
$$

after the following change of coordinates

$$
x=\frac{\left(q_{1}+q_{2}\right)^{2}}{4}, \quad p_{x}=\frac{p_{1}+p_{2}}{q_{1}+q_{2}}, \quad y=\frac{q_{1}-q_{2}}{2}, \quad p_{y}=p_{1}-p_{2},
$$

we can get superintegrable Stäckel system

$$
\begin{aligned}
& H_{1}=p_{x} p_{y}+\alpha y\left(x+y^{2}\right)+\beta\left(x+3 y^{2}\right)+\gamma y, \\
& H_{2}=\frac{p_{y}^{2}}{4}+p_{x}^{2} x-y p_{y} p_{x}+\frac{\alpha\left(3 y^{2}+x\right)\left(x-y^{2}\right)}{4}+2 \beta y\left(x-y^{2}\right)+\frac{\gamma}{2}\left(x-y^{2}\right),
\end{aligned}
$$


with the quadratic Euler integral

$$
K_{2}=s+c=\frac{p_{x}^{2}}{4}+\frac{\alpha y^{2}}{4}+\frac{\beta y}{2}
$$

and with the cubic integral of motion

$$
K_{3}=\sqrt{S}=\frac{p_{x}^{3}}{4}+\frac{\alpha\left(3 p_{x}\left(x+y^{2}\right)-p_{y} y\right)}{8}+\frac{\beta\left(6 p_{x} y-p y\right)}{8}+\frac{\gamma p_{x}}{8} .
$$

The corresponding classical trajectory of motion is defined by

$$
F(x, y)=H_{1} y+H_{2}-\frac{\alpha\left(x-y^{2}\right)^{2}}{4}+\beta y\left(x-y^{2}\right)+\frac{\gamma\left(x-3 y^{2}\right)}{6}=0 .
$$

The same system may be obtained by using fifth substitution from (3.7) as well.

Case 3 If we take the second solution from (3.7)

$$
u_{j}=q_{j}, \quad v_{j}=q_{j}^{2}, \quad \Rightarrow \quad p_{1}^{2} q_{1}^{2}=P\left(q_{1}^{2}\right), \quad p_{2}^{2} q_{2}^{2}=P\left(q_{2}^{2}\right)
$$

and quartic

$$
P(\lambda)=-\alpha \lambda^{4}-\frac{\beta}{2} \lambda^{3}+H_{1} \lambda^{2}+H_{2} \lambda+\gamma,
$$

after canonical transformations (3.13) we will get the following superintegrable Stäckel system

$$
\begin{aligned}
& H_{1}=p_{x} p_{y}+\alpha(x+3 y)(3 x+y)+\beta(x+y)+\frac{\gamma}{(x-y)^{2}}, \\
& H_{2}=\left(p_{x}-p_{y}\right)\left(p_{x} x-p_{y} y\right)-2 \alpha(x+y)(x-y)^{2}-\frac{\beta(x-y)^{2}}{2}-\frac{2 \gamma(x+y)}{(x-y)^{2}} .
\end{aligned}
$$

Using the Euler addition theorem (2.5) we can get the Euler integral

$$
K_{2}=s+c=\frac{\left(p_{x}+p_{y}\right)^{2}}{16}+\alpha(x+y)^{2}+\frac{\beta}{4}(x+y),
$$

the cubic in momenta integral of motion

$$
\begin{aligned}
K_{3}=\sqrt{S} & =\frac{\left(p_{x}-p_{y}\right)^{2}\left(p_{x}+p_{y}\right)}{32}-\frac{\alpha(x-y)\left(p_{x}(5 x+3 y)-(3 x+5 y) p_{y}\right)}{8} \\
& -\frac{\beta\left(p_{x}-p_{y}\right)(x-y)}{16}-\frac{\gamma\left(p_{x}+p_{y}\right)}{8(x-y)^{2}}
\end{aligned}
$$

and equation for the classical trajectory of motion

$$
F(x, y)=\left(y^{2}+x^{2}+\frac{2 x y}{3}\right) H_{1}+(x+y) H_{2}-\alpha(x-y)^{4}-\frac{\beta(x+y)(x-y)^{2}}{2}+\gamma=0 .
$$

This superintegrable system coincides with the one of the Drach systems associated with the logarithmic angle variables [15].

The same system may be obtained by using fourth substitution from (3.7).

Case 4 If we take the third solution from (3.7)

$$
u_{j}= \pm 1, \quad v_{j}= \pm q_{j}^{-1}, \quad \Rightarrow \quad p_{1}^{2}=X\left(q_{1}^{-1}\right), \quad p_{2}^{2}=Y\left(-q_{2}^{-1}\right)
$$

and quartic

$$
P(\lambda)=\alpha \lambda^{4}-\beta \lambda^{3}+H_{2} \lambda^{2}+H_{1} \lambda-\gamma,
$$


then after canonical transformation

$$
x=\sqrt{q_{1} q_{2}}, \quad p_{x}=\frac{p_{1} q_{1}+q_{2} p_{2}}{\sqrt{q_{1} q_{2}}}, \quad y=\frac{q_{1}-q_{2}}{\sqrt{q_{1} q_{2}}}, \quad p_{y}=\frac{\sqrt{q_{1} q_{2}}\left(p_{1} q_{1}-q_{2} p_{2}\right)}{q 1+q 2}
$$

we will get the following superintegrable Stäckel system

$$
H_{1}=p_{x} p_{y}+\frac{\alpha y}{x^{3}}+\frac{\beta}{x^{2}}+\gamma x y, \quad H_{2}=p_{y}^{2}+\frac{\left(p_{x} x-y p_{y}\right)^{2}}{4}-\frac{\alpha\left(y^{2}+1\right)}{x^{2}}-\frac{\beta y}{x}+\gamma x^{2} .
$$

Using the Euler addition theorem (2.10) one gets additional quadratic in momenta Euler integral

$$
K_{2}=s+C=\frac{\left(p_{x} x-y p_{y}\right)^{2}}{16}-\frac{\alpha y^{2}}{4 x^{2}}-\frac{\beta y}{4 x}
$$

the cubic integral of motion

$$
K_{3}=\sqrt{S}=\frac{p_{y}^{2}\left(p_{x} x-y p_{y}\right)}{8}+\frac{\alpha\left(3 y p_{y}+x p_{x}\right)}{8 x^{2}}+\frac{\beta p_{y}}{4 x}+\frac{\gamma\left(p_{x} x-y p_{y}\right) x^{2}}{8}
$$

and the following equation for classical trajectory of motion

$$
F(x, y)=-\frac{H_{1} y}{2 x}+\frac{H_{2}\left(y^{2}-2\right)}{6 x^{2}}+\frac{\alpha}{x^{4}}-\frac{\beta y}{2 x^{3}}-\gamma=0
$$

Case 5 Using the same third solution (3.7) and another quartic

$$
P(\lambda)=4 \alpha \lambda^{4}+4 \beta \lambda^{3}+H_{2} \lambda^{2}+2 \gamma \lambda+H_{1}
$$

after the following change of variables

$$
x=\frac{q_{1} q_{2}}{2}, \quad p_{x}=\frac{p_{1} q_{1}+q_{2} p_{2}}{q_{1} q_{2}}, \quad y=\frac{q_{1}^{2}+q_{2}^{2}}{2 q_{1} q_{2}}, \quad p_{y}=\frac{q_{1} q_{2}\left(p_{1} q_{1}-q_{2} p_{2}\right)}{q_{1}^{2}-q_{2}^{2}},
$$

we can get more complicated superintegrable system with the Stäckel integrals of motion

$$
\begin{aligned}
& H_{1}=p_{x} p_{y}+\frac{\alpha}{x^{2}}+\frac{\beta}{x^{3 / 2} \sqrt{y-1}}+\frac{\gamma}{x^{1 / 2} \sqrt{y-1}} \\
& H_{2}=\left(x p_{x}-p_{y}-p_{y} y\right)\left(x p_{x}+p_{y}-p_{y} y\right)-\frac{4 \alpha y}{x}-\frac{2 \beta(2 y-1)}{x^{1 / 2} \sqrt{y-1}}-\frac{2 \gamma x^{1 / 2}}{\sqrt{y-1}} .
\end{aligned}
$$

The Euler integral of motion is equal to

$$
K_{2}=s+c=\frac{\left(p_{x} x+p_{y}-p_{y} y\right)^{2}}{4}-\frac{\alpha(y-1)}{x}-\frac{\beta \sqrt{y-1}}{\sqrt{x}},
$$

the cubic in momenta integral reads as

$$
K_{3}=\sqrt{S}=-\frac{p_{y}\left(x p_{x}+p_{y}-p_{y} y\right)^{2}}{2}+\frac{2 \alpha(y-1) p_{y}}{x}-\frac{\beta\left(p_{x} x-3 p_{y} y+3 p y\right)}{2 x^{1 / 2} \sqrt{y-1}}-\frac{\gamma x^{1 / 2}\left(p_{x} x+p_{y}-p_{y} y\right)}{2 \sqrt{y-1}}
$$

and classical trajectory of motion is defined by the following equation

$$
F(x, y)=H_{1}+\frac{H_{2}(y-2)}{6 x}+\frac{\alpha}{x^{2}}-\frac{\beta \sqrt{y-1}}{x^{3 / 2}}+\frac{\gamma \sqrt{y-1}}{x^{1 / 2}} .
$$




\subsection{The quadratic integrals of motion}

It is easy to prove that the algebra of integrals of motion $H_{1,2}$ and $K_{2}$ is the quadratic Poisson algebra because

$$
\left\{H_{2}, K_{2}\right\}=\sigma K_{3}=\sigma \sqrt{S(s)}, \quad \text { where } \begin{cases}\sigma=2, & V_{1}, \\ \sigma=-2, & V_{2}, V_{4}, V_{5}, \\ \sigma=4, & V_{3},\end{cases}
$$

and

$$
\left\{H_{2}\left\{H_{2}, K_{2}\right\}\right\}=\left\{H_{2}, \sigma \sqrt{S}\right\}=\frac{\sigma^{2}}{2} S^{\prime}=\frac{\sigma^{2}}{2}\left(12 s^{2}-g_{2}\right)=\Phi\left(H_{1}, H_{2}, K_{2}\right),
$$

where $\Phi\left(H_{1}, H_{2}, K_{2}\right)$ is the second order polynomial such as $s=K_{2}-c$ and $g_{2}$ is quadrivariant of the quartic (2.8). Another details on the quadratic Poisson algebras of integrals of motion may be found in $[4]$.

The search of the two dimensional manifolds whose the geodesics are curves which possess two additional quadratic integrals of motion was initiated by Darboux [3], who found five classes of the metrics. These metrics or "formes essentielles" are tabulated in "Tableau" by Koenigs [10] and in [8].

The superintegrable Darboux-Koenigs systems have a generic conformal Hamiltonian

$$
H_{1}=\frac{p_{\xi} p_{\eta}}{\mathrm{g}(\xi, \eta)}+V(\xi, \eta)
$$

where the Darboux-Koenigs metric $g$ is a metric on the Liouville surface $[3,4]$ if

$$
\mathrm{g}(\xi, \eta)=F(\xi+\eta)+G(\xi-\eta), \quad \text { and } \quad K_{2}=p_{\xi}{ }^{2}+p_{\eta}{ }^{2}-2 p_{\xi} p_{\eta} \frac{\beta(\xi, \eta)}{\mathrm{g}(\xi, \eta)}+Q(\xi, \eta)
$$

or metric $g$ is a metric on the Lie surface if

$$
\mathrm{g}(\xi, \eta)=\xi F(\eta)+G(\eta), \quad \text { and } \quad K_{2}=p_{\xi}^{2}-2 p_{\xi} p_{\eta} \frac{\beta(\xi, \eta)}{\mathrm{g}(\xi, \eta)}+Q(\xi, \eta) .
$$

Superintegrable systems associated with the Liouville surfaces are separable in the two different orthogonal systems of coordinates. It means that two pairs of integrals of motion $\left(H_{1}, H_{2}\right)$ and $\left(H_{1}, K_{2}\right)$ take on the Stäckel form (3.3) after some different point transformations (3.8).

For the superintegrable systems associated with the Lie surfaces only one pair of integrals $\left(H_{1}, H_{2}\right)$ may be reduced to the Stäckel form (3.3), whereas second pair of integrals $\left(H_{1}, K_{2}\right)$ doesn't separable in the class of the point transformations (3.8).

It's easy to prove that two systems with potentials $V_{1}$ and $V_{2}$ are defined on the Lie surfaces. The remaining systems with potentials $V_{3}, V_{4}$ and $V_{5}$ are defined on the Liouville surfaces. The second separated variables $\widetilde{q}_{1,2}$ for integrals of motion $H_{1}, K_{2}$ may be found by using the software proposed in $[7]$ :

$$
\begin{aligned}
& x=\frac{\widetilde{q}_{1}^{2}-\widetilde{q}_{2}^{2}}{4}, \quad y=\frac{\widetilde{q}_{1}^{2}+\widetilde{q}_{2}^{2}}{4}, \quad \text { for } V_{3}, \\
& x=\widetilde{q}_{2} \widetilde{q}_{1}^{-2}, \quad y=\widetilde{q}_{2} \widetilde{q}_{1}^{2}, \quad \text { for } V_{4}, V_{5} .
\end{aligned}
$$

It is easy to prove that the corresponding separated relations $\widetilde{p}_{j}^{2}=P\left(\widetilde{q}_{j}\right)$ define two different zero-genus hyperelliptic curves and lead to logarithmic angle variables [15]. Thus, for these three Euler superintegrable systems there are two different addition theorems: addition theorem for elliptic functions (2.10) and addition theorem for logarithms $\ln x+\ln y=\ln x y$. So, multiseparability of the superintegrable systems may be associated with occurrence of the different addition theorems for a given superintegrable hamiltonian. 


\section{Conclusion}

In fact, Euler proposed construction of the algebraic integrals of motion for the equations

$$
\frac{\kappa_{1} d x_{1}}{\sqrt{P\left(x_{1}\right)}} \pm \frac{\kappa_{2} d x_{2}}{\sqrt{P\left(x_{2}\right)}}=0
$$

where $\kappa$ 's are integer. We discuss the Euler construction of the algebraic integrals of motion for superintegrable systems at $\kappa_{1,2}= \pm 1$ only. It will be interesting to classify the corresponding superintegrable systems for another values of $\kappa$ 's, because the corresponding additional integrals of motion will be higher order polynomials in momenta, see [15].

Another perspective consists in the substitution of the generic Darboux metrics into the equations (3.9) and classification of the corresponding Euler superintegrable systems on the Darboux spaces. It requires slightly more complicated calculations.

The research was partially supported by the RFBR grant 06-01-00140.

\section{References}

[1] W.S. Burnside and A.W. Panton, Theory of Equations, Longmans, 1886.

[2] A.G. Greenhill, The applications of elliptic functions, Macmillan and Co, London, 1892.

[3] G. Darboux, Leçons sur la théorie générale des surfaces et les applications geómétriques du calcul infinitésimal, v.1-4, Paris, Gauthier-Villars, 1887-89.

[4] C. Daskaloyannis, K. Ypsilantis, Unified treatment and classification of superintegrable systems with integrals quadratic in momenta on a two-dimensional manifold, J. Math. Phys., v.47, 042904, 2006.

[5] J. Drach. Sur l'intégration logique des équations de la dynamique à deux variables: Forces conservatives. Intêgrales cubiques. Mouvements dans le plan. Comptes Rendus, v.200, p.22-26, 1935.

[6] L. Euler, Institutiones Calculi integralis, Acta Petropolitana, 1761, (Russian translation GITL, Moskow, 1956.)

[7] Yu.A. Grigoryev, A.V. Tsiganov, Symbolic software for separation of variables in the HamiltonJacobi equation for the L-systems, Regular and Chaotic Dynamics, v.10(4), p.413-422, 2005.

[8] E.G. Kalnins, J.M. Kress, W. Miller Jr, Nondegenerate 2D complex Euclidean superintegrable systems and algebraic varieties, J. Phys. A: Math. Theor., 40, 3399-3411, 2007.

[9] E.G. Kalnins, J.M. Kress, G.S. Pogosyan and W. Miller, Completeness of superintegrability in two-dimensional constant-curvature spaces, J.Phys. A: Math. Gen. v.34, p.4705, 2001.

[10] M.G. Koenigs, Sur les géodésiques a intégrales quadratiques, Note II in G. Darboux, Leçons sur la Théorie Générale des Surfaces, 1898.

[11] F. Richelot, Ueber die Integration eines merkwürdigen Systems von Differentialgleichungen, J. Reine Angew. Math. v.23, p.354-369,1842.

[12] P. Stäckel Uber die Integration der Hamilton-Jacobischen Differential Gleichung Mittelst Separation der Variabel, Habilitationsschrift, Halle, 1891.

[13] A.V. Tsiganov, The Stäckel systems and algebraic curves, J. Math. Phys., v.40, p.279-298, 1999.

[14] A.V. Tsiganov, Duality between integrable Stäckel systems, J. Phys.A: Math. Gen., v.32, p.79657982, 1999.

[15] A.V. Tsiganov, Addition theorem and the Drach superintegrable systems, J. Phys. A: Math. Theor., v.41(33), 335204 (16pp), 2008.

[16] K. Weierstrass, Math. Werke, v.I, Berlin, Mayer and Müller, 1895. 